\title{
Different influence of pyrimidine and benzene rings on current-voltage characteristics of molecular devices
}

\author{
Xiao-Xiao $\mathrm{Fu}$ and Zong-Liang $\mathrm{Li}^{*}$ \\ College of Physics and Electronics, Shandong Normal University, Jinan 250014, \\ China
}

Received 1 April 2011; Accepted (in revised version) 28 April 2011

Published Online 8 November 2011

\begin{abstract}
By using $a b$ initio method and elastic scattering Green's function theory, the electronic transport properties of symmetric tetraphenyl and non-symmetric diblock dipyrimidinyldiphenyl molecules covalently bonded to two electrodes are investigated. The numerical results show that, the tetraphenyl molecule has better electronic conductivity than the diblock molecule. The latter exhibits pronounced rectification behavior. Those molecular orbitals of the tetraphenyl molecule delocalized into two gold electrodes simultaneously result in better electronic conductivity. However, the non-symmetric structure of the diblock molecule leads to the localization of molecular orbitals, which is a disadvantage to electronic transport.
\end{abstract}

PACS: 31.10.+z, 31.15.-p, 31.15.A-, 31.15.es

Key words: tetraphenyl molecule, dipyrimidinyldiphenyl molecule, elastic scattering Green's function, current-voltage characteristic

\section{Introduction}

In recent years, due to the continuous improvement and widespread application of singlemolecule technology, molecular electronics has developed rapidly and achieved encouraging results both in experiment and theory [1-13]. In the 1980s, the scanning tunneling microscope (STM), fluorescence probe method and optical tweezers appeared, subsequently the atomic force microscope (AFM), electrostatic force microscope, scanning particle conductance microscope, and scanning tunneling spectroscope (STS) emerged, form a rich scanning probe system. The emergence of these single molecule manipulation techniques makes the single-molecule studies no longer on paper. In experiment, people applied these techniques to create a series of molecular devices with special features successfully, such as molecular wires, molecular rectifiers [2], molecular switches [3], molecular memories and molecular

\footnotetext{
${ }^{*}$ Corresponding author. Email address: lizongliang@sdnu .edu .cn (Z. L. Li)
} 
motors [5]. With the progress of experimental work, theoretical staffs developed a variety of methods to simulate the working mechanism of molecular devices and research the relationship between molecular structure and electronic transport properties of them. It was discovered that the electronic transport properties of molecules are related to not only the molecular electronic structure but also the interaction between the molecule and metal electrodes, such as the mode of the molecule coupled to the electrodes, the molecular orientation on the metal surface [14], the type of electrodes, the distance of two electrodes [15-17], electrode force, electrode dimensions [18]. In addition, temperature and solvent effect [19], etc. also have some effect on the molecular electronic transport properties.

The functional molecules including the conjugated molecule composed of $\pi$ bond, the saturated alkyl thiol chain formed by $\sigma$ bond, single or multi-layer carbon nanotubes, Fullerenes, and bio-organic molecules (e.g. DNA molecules) have been paid much attention to in research. Since the molecules containing benzene ring possess rich $\pi$ electrons which are beneficial to electronic transport, the aromatic compounds in which the benzene ring exists as a basic structural unit, are of great concern in the study. Containing nitrogen atoms or sulfur atoms, the heterocyclic system similar to the benzene ring is also rich in $\pi$-electrons, and therefore a good choice for molecular devices. However, in previous studies, people paid more attention to molecules with symmetrical geometry. In fact, asymmetric molecules formed by $\pi$-electron systems of different structures, have more abundant non-linear electronic transport properties than symmetrical ones [20-22]. Recently, Yu synthesized dipyrimidinyldiphenyl molecules with benzene and heterocyclic pyrimidine as the basic unit in experiment, finding that the asymmetry of the molecular geometric structure results in obvious rectification property [23]. In order to understand the transport properties of two different molecules better, we use density functional theory based on elastic scattering Green function approach to investigate the two kinds of molecules theoretically. The different effects of pyrimidine and benzene rings on the optimized molecular structure, current-voltage characteristic, molecular orbital and coupling coefficient, are discussed.

\subsection{Theoretical model and numerical method}

The organic molecule is sandwiched between two electron reservoirs (the source and the drain, abbreviated as $S$ and $D$ ). For a three-dimensional electrode, when an external bias is applied, the net current of the device from the source to the drain can be written as

$$
\begin{aligned}
I_{S D}=\left(\frac{9 \pi}{4}\right)^{\frac{1}{3}} \frac{9 e k_{B} T}{2 h E_{f}^{2}} \int\{ & \ln \left[1+\exp \left(\frac{E_{f}+e V_{S D}-E_{z}}{k_{B} T}\right)\right] \\
& \left.-\ln \left[1+\exp \left(\frac{E_{f}-E_{z}}{k_{B} T}\right)\right]\right\}\left|T\left(E_{z}, V_{S D}\right)\right|^{2} \frac{d E_{z}}{E_{z}},
\end{aligned}
$$


where $E_{f}$ is Fermi level, and $V_{S D}$ is the external bias. In Eq. (1), the transition matrix element can be determined by

$$
T\left(E, V_{S D}\right)=\sum_{J} \sum_{K} Y_{J S}\left(V_{S D}\right) Y_{D K}\left(V_{S D}\right) \sum_{n} \frac{\langle J \mid n\rangle\langle n \mid K\rangle}{\left(E-E_{n}(V)\right)+i \Gamma_{n}},
$$

where $Y_{J S}$ and $Y_{D K}$ represent the couplings between each atom of the molecule and the gold clusters (noted by $S$ and $D$ ), $|n\rangle$ is the eigenstate of the Hamiltonian of the composite goldmolecule-gold system, $E$ is the energy at which the scattering process is observed, $E_{n}(V)$ is the eigenvalue of the system, and $\Gamma_{n}$ is the energy broadening.

Taking into account that only the nearest atoms have evident interaction with the molecules, we used two gold clusters to simulate the interaction between gold electrodes and molecules. In the numerical simulation, each gold cluster consists of three gold atoms with equilateral triangle configuration. The Au-Au bond length is fixed to be $0.288 \mathrm{~nm}$. According to the energy of the system, the terminal sulfur atoms of the molecules are set on the hollow positions of the gold triangles. The molecules sandwiched between gold electrodes compose extended molecular systems (see Fig. 1). The geometry of the extended molecules was optimized on the Gaussian03 package [24], by means of the hybrid density functional theory (Becke3LYP) method, with LanL2DZ as the basis set. Electronic transport properties of the molecular devices were calculated with QCME package [25].

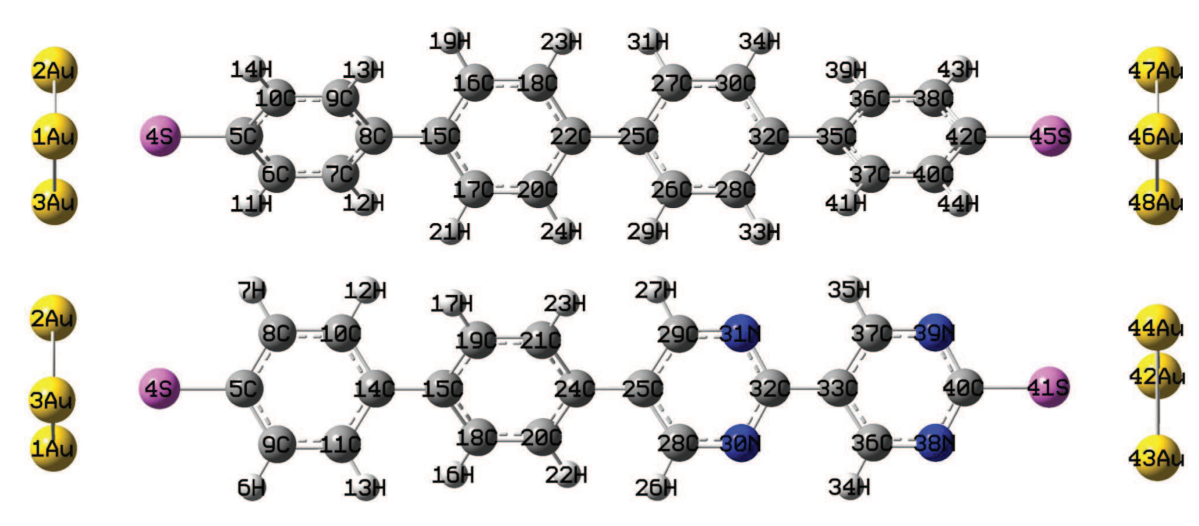

Figure 1: Structure of the extended molecules and arrangement of all atoms.

\section{Results and discussion}

\subsection{Geometric structures of the molecular junctions}

The geometric optimization shows that, in the tetraphenyl extended molecule, the distance of two gold electrodes at both ends of the molecule is $2.43 \mathrm{~nm}$, and four benzene rings are 
not in one plane, but have a certain twist angle between each other. However, in the dipyrimidinyldiphenyl extended molecule, that distance is $2.40 \mathrm{~nm}$, and there is a certain angle both between two benzene planes and between the adjacent benzene and pyrimidine planes, while the two pyrimidine rings are approximately in one plane. In traphenyl molecule, $\mathrm{H}$ atoms on benzene rings stand close to each other, whose electron clouds overlap largely, resulting in significant Coulomb repulsion, so that the torsion angle between neighboring benzene rings occurs. In dipyrimidinyldiphenyl molecule, since the nitrogen atoms on pyrimidine rings connect with no hydrogen atoms, the lone electron pairs of nitrogen atoms on one pyrimidine ring develop affinity with the hydrogen atoms on the other one, therefore the two neighboring pyrimidine rings are almost in one plane.

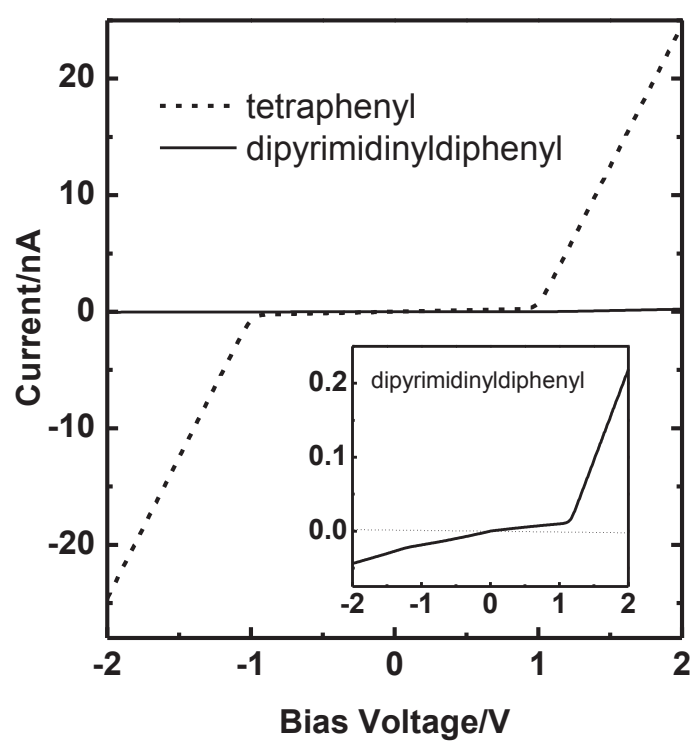

Figure 2: Current-voltage characteristic of the tetraphenyl and dipyrimidinyldiphenyl molecule.

\subsection{Current-voltage characteristics of the molecular junctions}

The current through the traphenyl and dipyrimidinyldiphenyl molecular junctions is shown in Fig. 2. The positive direction of current is defined to flow from the benzene ring to pyrimidine ring. The current-voltage characteristic curve of traphenyl molecules indicates that when the absolute value of applied bias is less than $1.0 \mathrm{~V}$, the current is very weak, while it is greater than $1.0 \mathrm{~V}$, the current increases rapidly with the increasing bias. From Fig. 2 we can see easily that, in the bias voltage region $[-2 \mathrm{~V}, 2 \mathrm{~V}]$, the current of dipyrimidinyldiphenyl molecules is much weaker than that of traphenyl molecules. For example, at the bias voltage of $2 \mathrm{~V}$, the current that flows through the traphenyl is $25 \mathrm{nA}$, larger than that through the dipyrimidinyldiphenyl (only about $0.2 \mathrm{nA}$ ) by two orders of magnitude. Thus, compared 
with the $\mathrm{C}$ atom on benzene ring, the nitrogen atom on pyrimidine ring throws significant impediment to the electronic transport of molecules.

However, from the illustration of Fig. 2, one can find that the transport properties of dipyrimidinyldiphenyl molecules are visibly different from those of traphenyl molecules. Especially in the bias voltage region $[1 \mathrm{~V}, 2 \mathrm{~V}]$, electric conductivity of the dipyrimidinyldiphenyl molecule under positive bias is evidently greater than that under negative bias, which shows so-called rectification characteristic. The figure shows that, at $2.0 \mathrm{~V}$ and $-2.0 \mathrm{~V}$ bias, the current flowing though dipyrimidinyldiphenyl molecules is $0.219 \mathrm{nA}$ and $-0.0439 \mathrm{nA}$, respectively, and the ratio of them (rectification ratio) is about $5: 1$, which agrees well with the experimental measured results. Obviously, it is the asymmetry of elements on benzene ring and pyrimidine ring in dipyrimidinyldiphenyl molecules that causes the rectification behavior. Because of its asymmetric structure, both the geometric structure and electronic structure have different responses to positive and negative bias, resulting in the asymmetry of electronic transport properties. The electric conductivity of tetraphenyl under positive and negative bias is almost symmetric.

\subsection{Electronic structures of the molecular junctions}

Electronic transport properties of molecules have close relationship with electronic structure of molecules. In order to understand the distinct electronic transport properties of the tetraphenyl and dipyrimidinyldiphenyl molecules better, we exhibit four molecular orbitals (LUMO, LUMO+1, LUMO+2 and LUMO+3) in Fig. 3. It is found that the LUMO of tetraphenyl molecules is mainly localized at the region of the molecule contacted with the right electrode, while the left electrode does not contribute to the molecular orbital, thereby LUMO makes almost no contribution to conductivity. However, the other three molecular orbitals (LUMO+1, $\mathrm{LUMO}+2$ and LUMO+3) of tetraphenyl molecules are delocalized well, which distribute to not only both ends of the molecule, but also the interior, making the molecule couple well with both electrodes through these orbitals. Due to the contribution of these orbitals, electrons in one electronic reservoir are easy to access to the molecule, flow from one side of the molecule to the other side, and then remove from the other side to the electrode, bringing resonant tunneling ultimately. Different from the molecular orbitals of tetraphenyl molecules, those of dipyrimidinyldiphenyl molecules couple with only one end of the electrodes, therefore, there are few resonant tunneling electrons. In terms of the orbital energy, under low voltage, the electron transport is mainly determined by these molecular orbitals, so it inevitably leads to better conductivity of tetraphenyl molecules than dipyrimidinyldiphenyl molecules. Relative to the Fermi level of the system, the energy of the three molecular orbitals (LUMO+1, LUMO +2 and LUMO+3) of tetraphenyl molecules is about $1.0 \mathrm{eV}$. From Fig. 2, it is easily found that because of their contribution, the current of tetraphenyl molecules begins to grow rapidly with bias voltage around $1.0 \mathrm{~V}$. As for dipyrimidinyldiphenyl molecules, the energy of these four molecular orbitals relative to the Fermi level is about $1.2 \mathrm{eV}$. Although the large portions of these four orbitals are localized at the one end of the molecular system and only a few proportions spread into the interior of the molecule, they still make some contribu- 
tion to the electronic transport. Moreover, owing to the asymmetric of dipyrimidinyldiphenyl molecules, the molecular orbitals have different responses to positive and negative bias, so that the electric conductivity of dipyrimidinyldiphenyl molecules becomes asymmetry under the positive and negative bias, resulting in rectification behavior.
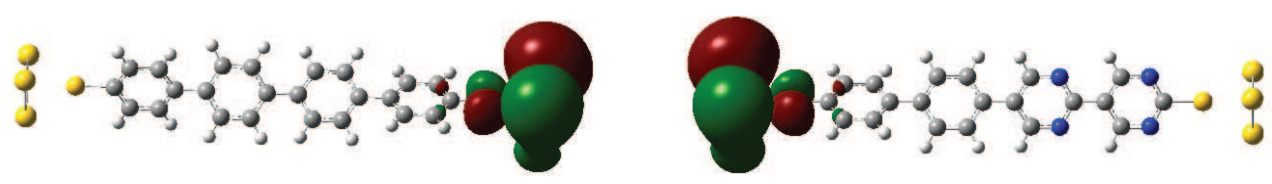

(a)
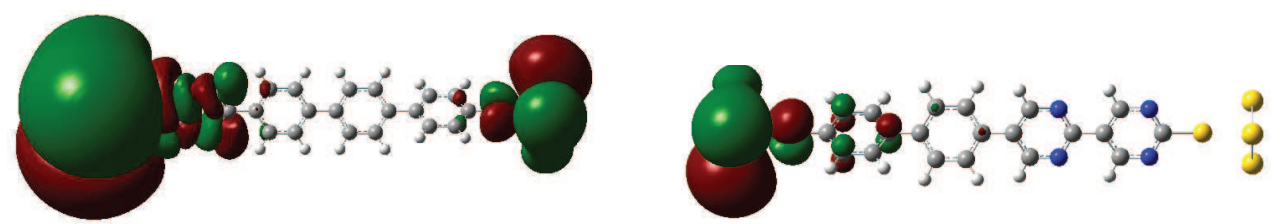

(b)
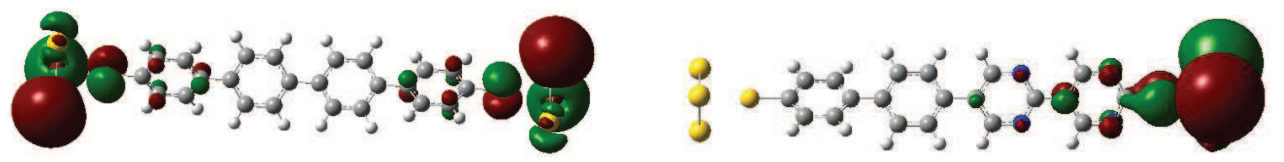

(c)
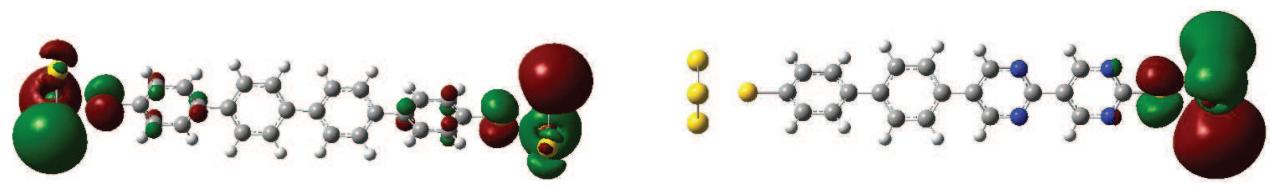

(d)

Figure 3: Molecular orbitals of the tetraphenyl molecule (left) and dipyrimidinyldiphenyl molecule (right). (a)-(d) corresponds to the orbital of LUMO, LUMO+1, LUMO+2 and LUMO+3, respectively.

\subsection{Coupling coefficients of the molecular junctions}

Eq. (2) indicates that the coupling coefficient between molecules and electrodes is one important factor affecting electronic transport properties of molecules. Tables 1 and 2 show the coupling coefficients between the atoms that couple strongly with the electrodes and the gold atoms. For dipyrimidinyldiphenyl molecules (Table 1), during the voltage ranges from $2 \mathrm{~V}$ to $-2 \mathrm{~V}$, the absolute values of coupling coefficients between the left electrode and the $\mathrm{S}, \mathrm{C}$ atoms near the left side decrease; on the contrary, those between the right electrode and the $\mathrm{S}, \mathrm{C}, \mathrm{N}$ atoms near the right side increase, among which the change of bias voltage produces a significant impact on the coupling coefficients between the electrodes and $\mathrm{S}$ atoms. It can 
be found as well that the coupling coefficient between C5 atom on the left benzene ring and the left electrode is higher than that between $\mathrm{C} 40$ atom at symmetrical positions on the right pyrimidine ring and the right electrode, and the latter has obvious responses to bias. By analysis of coupling coefficients, we assure that the impact of external bias on pyrimidine and benzene rings is asymmetrical. For tetraphenyl molecules, the data in Table 2 show that, over the bias changing from $2 \mathrm{~V}$ to $-2 \mathrm{~V}$, the coupling coefficients almost remain unchanged, moreover, the coupling coefficients of the left and right ends are of symmetry generally. Therefore, the asymmetric responses of dipyrimidinyldiphenyl molecules to positive and negative bias is also a substantial cause for rectifying properties of molecules.

Table 1: Coupling coefficients of the dipyrimidinyldiphenyl molecule.

\begin{tabular}{|c|cccc|cccc|}
\hline & \multicolumn{4}{|c|}{$\begin{array}{c}\text { Coefficients of the atoms coupling } \\
\text { with the left electrode }\end{array}$} & \multicolumn{4}{c|}{$\begin{array}{c}\text { Coefficients of the atoms coupling with } \\
\text { the right electrode }\end{array}$} \\
\cline { 2 - 9 } & S4 & C5 & C8 & C9 & N38 & N39 & C40 & S41 \\
\hline 2V & $6.10 \mathrm{E}-1$ & $-4.59 \mathrm{E}-1$ & $7.53 \mathrm{E}-2$ & $9.09 \mathrm{E}-2$ & $-9.64 \mathrm{E}-2$ & $-9.04 \mathrm{E}-2$ & $-9.68 \mathrm{E}-2$ & $3.68 \mathrm{E}-1$ \\
0V & $4.95 \mathrm{E}-1$ & $-4.35 \mathrm{E}-1$ & $7.01 \mathrm{E}-2$ & $8.91 \mathrm{E}-2$ & $-9.91 \mathrm{E}-2$ & $-8.98 \mathrm{E}-2$ & $-1.25 \mathrm{E}-1$ & $5.19 \mathrm{E}-1$ \\
-2V & $3.65 \mathrm{E}-1$ & $-4.03 \mathrm{E}-1$ & $6.80 \mathrm{E}-2$ & $8.41 \mathrm{E}-2$ & $-9.94 \mathrm{E}-2$ & $-9.31 \mathrm{E}-2$ & $-1.45 \mathrm{E}-1$ & $6.56 \mathrm{E}-1$ \\
\hline
\end{tabular}

Table 2: Coupling coefficients of the tetraphenyl molecule.

\begin{tabular}{|c|cccc|cccc|}
\hline \multirow{2}{*}{} & \multicolumn{4}{|c|}{$\begin{array}{c}\text { Coefficients of the atoms coupling } \\
\text { with the left electrode }\end{array}$} & \multicolumn{4}{c|}{$\begin{array}{c}\text { coefficients of the atoms coupling } \\
\text { with the right electrode }\end{array}$} \\
\cline { 2 - 9 } & S4 & C5 & C6 & C10 & C38 & C40 & C42 & S45 \\
\hline 2V & $5.00 \mathrm{E}-1$ & $-4.19 \mathrm{E}-1$ & $6.95 \mathrm{E}-2$ & $8.41 \mathrm{E}-2$ & $6.96 \mathrm{E}-2$ & $8.40 \mathrm{E}-2$ & $-4.19 \mathrm{E}-1$ & $5.00 \mathrm{E}-1$ \\
0V & $5.01 \mathrm{E}-1$ & $-4.20 \mathrm{E}-1$ & $6.93 \mathrm{E}-2$ & $8.49 \mathrm{E}-2$ & $6.95 \mathrm{E}-2$ & $8.49 \mathrm{E}-2$ & $-4.21 \mathrm{E}-1$ & $5.01 \mathrm{E}-1$ \\
$-2 \mathrm{~V}$ & $4.99 \mathrm{E}-1$ & $-4.19 \mathrm{E}-1$ & $6.88 \mathrm{E}-2$ & $8.54 \mathrm{E}-2$ & $6.89 \mathrm{E}-2$ & $8.52 \mathrm{E}-2$ & $-4.19 \mathrm{E}-1$ & $4.99 \mathrm{E}-1$ \\
\hline
\end{tabular}

\section{Conclusion}

Based on the hybrid density functional method and the elastic scattering Green function theory, the electronic transport properties of dipyrimidinyldiphenyl and tetraphenyl molecules are researched theoretically. It is found that three molecular orbitals (LUMO+1, LUMO+2 and LUMO+3) of tetraphenyl molecules are delocalized in a certain extent, resulting in better conductive properties than dipyrimidinyldiphenyl molecules. As for dipyrimidinyldiphenyl molecules, composed of the two blocks, the difference of elements on pyrimidine and benzene ring makes the molecular orbitals that are decisive of electrical transport so localized as to be unfavorable to electronic transport. In addition, the difference of elements composing two block of dipyrimidinyldiphenyl is the fundamental reason for the asymmetry of $I-V$ characteristic curves or good rectification properties, which conclusion is in good agreement with the experimental results. 
Acknowledgments. This work is supported by the National Natural Science Foundation of China under Grant Nos. 10804064 and 10974121.

\section{References}

[1] J. Chen, W. Wang, M. A. Reed, et al., Appl. Phys. Lett. 77 (2000) 1224.

[2] J. O. Lee, G. Lientschnig, F. Wiertz, et al., Nano Lett. 3 (2003) 113.

[3] A. Nitzan and M. A. Rater, Science 300 (2003) 1384.

[4] D. M. Cardamone, C. A. Stafford, and S. Mazumdar, Nano Lett. 6 (2006) 2422.

[5] N. J. Tao, Nat. Nanotechnol. 1 (2006) 173.

[6] H. B. Akkerman, P. W. M. Bloom, D. M. deLeeuw, et al., Nature 441 (2006) 69.

[7] A. Saraiva-Souza, B. G. Sumpter, V. Meunier, et al., J. Phys. Chem. C 112 (2008) 12008.

[8] J. L. Pitters and R. A. Wolkow, Nano Lett. 6 (2006) 390.

[9] S. Roy, H. Vedala, A. D. Roy, et al., Nano Lett. 8 (2008) 26.

[10] Y. F. Ye, M. L. Zhang, and J. W. Zhao, J. Mol. Struct. (Theochem.) 822 (2007) 12.

[11] S. Lakshmi, S. Dutta, and S. K. Pati, J. Phys. Chem. 112 (2008) 14718.

[12] R. J. Liu, X. W. Yan, Z. L. Li, et al., J. At. Mol. Phys. 24 (2007) 1041 (in Chinese).

[13] Z. L. Li, B. Zou, X. W. Yan, et al., Chinese Phys. 16 (2007) 1434.

[14] C. J. Xia, C. F. Chang, G. C. Hu, et al., Acta Phys. Sin. 56 (2007) 4884 (in Chinese).

[15] R. J. Liu, Z. L. Li, X. W. Yan, et al., J. At. Mol. Phys. 25 (2008) 519 (in Chinese).

[16] B. Zou, Z. L. Li, C. K. Wang, et al., Acta Phys. Sin. 54 (2005) 1341 (in Chinese).

[17] B. Zou, Z. L. Li, X. N. Song, et al., Acta Phys. -Chim. Sin. 23 (2007) 1577 (in Chinese).

[18] Z. L. Li, C. K. Wang, Y. Luo, et al., Acta Phys. Sin. 53 (2004) 1490 (in Chinese).

[19] Z. L. Li, B. Zou, and C. K. Wang, Phys. Rev. B 73 (2006) 075326.

[20] J. Huang, Q. X. Li, Z. Y. Li, et al., J. Nanosci. Nanotechnol. 8 (2008) 1.

[21] G. M. Morales, P. Jiang, S. Yuan, et al., J. Am. Chem. Soc. 127 (2005) 10456.

[22] Z. L. Gasyna, G. M. Morales, A. Sanchez, et al., Chem. Phys. Lett. 417 (2006) 401.

[23] I. Díez-Pérez, J. Hihath, Y. Lee, et al., Nature Chem. 1 (2009) 635.

[24] M. J. Frisch, G. W. Trucks, H. B. Schlegel, et al., Gaussian 03, Rev. D.1 (Gaussian, Inc., Pittsburgh PA, 2003).

[25] J. Jiang, C. K. Wang, and Y. Luo, QCME-V1.1, Quantum Chemistry for Molecular Electronics (Royal Institute of Technology, Sweden, 2006). 